\title{
Seed-based System for Cost-Effective Production of Vaccine Against Chronic Respiratory Disease in Chickens
}

\author{
Imran Khan ${ }^{1}$, Peyman Habibi ${ }^{2}$, Madiha Saeed ${ }^{3}$, Ayesha Haq ${ }^{3}$, and Yao Shi ${ }^{1}$ \\ ${ }^{1}$ University of Pennsylvania \\ ${ }^{2}$ University of Pennsylvania Perelman School of Medicine \\ ${ }^{3}$ International Islamic University
}

March 22, 2021

\begin{abstract}
The production of vaccines in plant cells, termed as plant-made pharmaceuticals or molecular farming, is a promising technology. Compared to mammalian cell lines, like HEK293 and CHO as established platform, plants can be grown cost effectively on a large-scale without necessitating any sophisticated technologies. An innovative application of this alternative system is the production of vaccines in edible tissues that can be consumed orally to deliver protein antigen without any further processing. In this project we reported stable expression of TM-1 protein of MG as vaccine candidate antigen against Chronic Respiratory Disease (CRD) in wheat seeds tissues. The molecular and Immunoblotting analysis confirmed the integration of recombinant protein of $41.8 \mathrm{kDa}$ with expression level of $1.03 \mathrm{mg} / \mathrm{g} \mathrm{DW}$ in endosperm tissues. When orally delivered, the plant made vaccine were highly effective in terms of developing antibodies in chicken without any detectable weight loss. Two doses of orally delivered plant-made TM-1 vaccine candidate elicited an immune response and protective effect against MG virus challenge at the level comparable to commercially available inactivated vaccine against CRD. Our study demonstrated that plant-made vaccines are not only safe but also similarly effective to commercially available vaccines.
\end{abstract}

Seed-based System for Cost-Effective Production of Vaccine Against Chronic Respiratory Disease in Chickens

Imran Khan ${ }^{1}$, Peyman Habibi ${ }^{2}$, Ayesha Naveed Ul Haq ${ }^{3}$, Madiha Saeed ${ }^{3}$, Yao Shi ${ }^{1 *}$

${ }^{1}$ Department of Basic and Translational Sciences, School of Dental Medicine, University of Pennsylvania, Philadelphia, PA, USA.

${ }^{2}$ Department of Pathology and Laboratory Medicine and Center for Cellular Immunotherapies, Perelman School of Medicine, University of Pennsylvania, Philadelphia, Pennsylvania 19104, USA.

${ }^{3}$ SA-Center for Interdisciplinary Research in Basic Sciences, International Islamic University, Islamabad, Pakistan

${ }^{*}$ Corresponding Author:

Yao Shi

School of Dental Medicine,

University of Pennsylvania,

240 South 40th Street,

547 Levy Building 
Philadelphia PA 19104-6030

Email: yaoshi@upenn.edu

Phone: 414-520-6920

Running title : Seed-based oral vaccine against Chronic Respiratory Disease in chickens

Key words : Molecular farming, oral vaccine, Chronic Respiratory Disease, immunization, seed-based expression

Abbreviations : CTB, cholera toxin B subunit; MG,Mycoplasma gallisepticum ; CRD, Chronic Respiratory Disease; PDs, Protein drugs.

\begin{abstract}
The production of vaccines in plant cells, termed as plant-made pharmaceuticals or molecular farming, is a promising technology. Compared to mammalian cell lines, like HEK293 and CHO as established platform, plants can be grown cost effectively on a large-scale without necessitating any sophisticated technologies. An innovative application of this alternative system is the production of vaccines in edible tissues that can be consumed orally to deliver protein antigen without any further processing. In this project we reported stable expression of TM-1 protein of MG as vaccine candidate antigen against Chronic Respiratory Disease (CRD) in wheat seeds tissues. The molecular and Immunoblotting analysis confirmed the integration of recombinant protein of $41.8 \mathrm{kDa}$ with expression level of $1.03 \mathrm{mg} / \mathrm{g} \mathrm{DW}$ in endosperm tissues. When orally delivered, the plant made vaccine were highly effective in terms of developing antibodies in chicken without any detectable weight loss. Two doses of orally delivered plant-made TM-1 vaccine candidate elicited an immune response and protective effect against MG virus challenge at the level comparable to commercially available inactivated vaccine against CRD. Our study demonstrated that plant-made vaccines are not only safe but also similarly effective to commercially available vaccines.
\end{abstract}

\title{
Introduction
}

Plants can be an attractive system to produce immunogens to control different human and veterinary diseases. The idea of utilizing plants as bioreactor for the expression of veterinary vaccines has been studied extensively in the last decade. Progress in plant genetic engineering has opened novel opportunities to use plants tissues as bioreactors for safe and cost-effective production of vaccine antigens. Plants provide an alternative expression system to microbes or mammalian cells grown in fermenters, which have limited capacity, higher production costs and in case of bacteria, lack of post translational modifications. Moreover, molecular farming has an edge over other production systems for recombinant proteins in terms of risk of the contamination with human and animal pathogen, microbial toxins, or oncogenic sequences. In the last two decades, plants have been used as viable and efficient bioreactor for the large-scale production of recombinant proteins including pharmaceutical proteins, antibodies [1], hormones, replacement enzymes [2], industrial enzymes [3] as well as vaccine antigens against infectious diseases [4]. Till now, variety of vaccines against veterinary diseases have been expressed using plant tissues as bioreactor [5]. The proof-of-concept for such studies has already been accomplished in previous studies and plants have been found suitable host for expression of edible vaccines for Newcastle disease [6], influenza, Ebola, infectious bronchitis virus (IBV), infectious bursal disease virus (IBDV) in chickens [7]. The plant made ZMapp antibodies against Ebola and influenza were not only encouraging but also paved a way for plants to be further utilized as bioreactor to combat infectious diseases in cost effective manner. Plant expressing vaccines for poultry against Newcastle disease virus has already been approved by USDA in 2006 [8] where vaccine development for animals was intensified, therefore, regulatory approval of plant made vaccines against veterinary disease can be achieved following same regulatory pathway.

Worldwide, poultry is the largest livestock group, and according to estimates with market value of $\$ 309$ billion in 2019 and expected to grow nearly 6\% annually till 2023 (Poultry world, 2019). The most prevalent problem in poultry especially at large scale setup includes zoonotic diseases which not only is threat to poul- 
try producers but also is a great risk for poultry genetic resources [6]. Thus, suitable veterinary strategies against such diseases are in crucial need of poultry setups. At present, there are occurrences of Chronic Respiratory Disease (CRD) worldwide and possess implacably drastic effects on small-scale poultry producers in underdeveloped countries. Mycoplasma gallisepticum (MG) is a bacteria-like organism that causes respiratory disease primarily in chickens and turkeys, that can spread both vertically and horizontally, that and can also infect gamebirds, pigeons, ducks, geese, peafowl, and wild birds. Symptoms of CRD includes stress caused by moving the birds, by de-beaking, operations handlings or unfavorable conditions (bad ventilation or cold). At present, poultry management activities such as feeding and watering, treatment with herbs, antibiotics, vaccines, and cleaning poultry houses are mainly carried out against diseases [9]. The use of antibiotics is one of many important tools in the treatment of poultry diseases. Use of antibiotics has brought unintended impacts on poultry production industries, such as the increase of infections in animals and the decrease of animal production. Antibiotics residues can remain in body for long time and may also greatly reduce scavenger wildlife populations. The potential relationship between circulating antibiotic residues and the presence of bacterial and fungal pathogens causing severe diseases. To overcome these problems, an innovative strategy is to develop cost effective vaccines against poultry diseases, mainly formed through molecular farming is urgently required because vaccination offers more long-term protection and prevention against diseases in poultry. Vaccination also enhance the immune system, without affecting the normal bacteria in body and can easily be decompose. Plant based vaccines should also be encouraged as they can easily be manufactured and less expensive as compared to other vaccines and antibiotics.

In this study, in view of mounting production cost for veterinary vaccines, we expressed candidate antigen vaccine against $\mathrm{CRD}$ in wheat seeds as promising model for cost effective vaccine development in plants that can be delivered orally without requiring any purification or downstream processing. The plant's seeds have naturally evolved system to express and accumulate storage proteins in large amount up to $40 \%$ of their weight [10]. The accumulation of such large amount of proteins distinguish seed over green tissues and tubers, where lower amount of proteins expression can be found [11]. Protein stability in seeds tissues for longer duration at ambient temperature is huge advantage where antibodies and vaccine antigens expressed in higher amount remain functional and stable for several years at room temperature $[10,12,13]$.

We also demonstrate the feasibility of developing plant based orally delivered vaccines as cost effective alternative. The oral delivery of vaccine candidate has been reported earlier in numerous studies [14-16]. The downstream processing that accounts for more than $80 \%$ of total production cost can be eliminated if the vaccine can be delivered orally without necessitating further processing. The oral delivery of protein drugs (PDs) is facilitated by plant cell wall that contains $\beta-1,4$ and $\beta-1,6$ linkages resistant to hydrolysis and allows PDs to be functionally active without any degradation during passing through enzymatic conditions in stomach. Further, certain gut bacteria that have the ability to hydrolyze plant cell wall contributes to release of PDs and its absorption into blood stream.

In this study, we stably engineered TM-1 protein as promising candidate antigen in wheat seeds tissues. The vaccine candidate was orally administered in chickens produced antibodies those able to inhibit infections after second drug dosage. Our findings demonstrate that oral delivery of vaccine is a promising approach that can revolutionize current production and delivery system for safe and affordable vaccine development.

\section{Materials and methods}

\subsection{Construct development and wheat transformation}

The binary vector pPZP211 vector was purchased commercially (Creative Biogene). A rice endospermspecific promoter GluB-4 [17] and a Nos terminator (GenBank: AJ007623 ) were inserted upstream and downstream of the multiple cloning site (MCS) atEcoR I/Kpn I and Xba I/Hind III sites, respectively. The codon-optimized complete CDS of TM-1 protein of Mycoplasma gallisepticum (MG; GenBank: E09896 ) was followed by the endoplasmic reticulum-retention signal KDEL (Lys-Asp-Glu-Leu) linked to C-terminal of TM-1. The CTB (cholera toxin B subunit) coding sequence followed by a furin enzymatic cleavage site (RRKRSV) were inserted immediately upstream of TM-1. The entire PGluB-4-CTB-furin-TM-1-KDEL- 
Tnos cassette was synthesized (GenScript, NJ, USA) and subcloned in pPZP211 at Kpn I/Xba I sites (Figure 1A).

Wildtype wheat (Triticum aestivum L. cv Bobwhite) was used to generate transgenic plants in this study. PDS-1000/He particle gun or biolistics (Biorad, Germany) was utilized for direct bombardment. The 20ug plasmid DNA was coated with gold particles of size $(0.6 \mu \mathrm{m})$ following procedure as described by Sanford et al., 1993, and with 950 psi rapture disks for bombardment. The immature embryos were isolated 14 days after anthesis (size $0.5-1.5 \mathrm{~mm}$ ) under aseptic conditions and were placed on induction media for 5-6 days in dark at $26 \mathrm{C}$ before bombardment for callus induction.

The total DNA was extracted from wheat seeds for confirmation of genetic integration of PGluB-4-TM-1-Tnos construct in wheat seed genome by Polymerase Chain Reaction (PCR) and sequencing with primers targeting transgene TM-1 (Seq Forward: 5'-attgcaaagctacctttttctatta-3'; Seq Reverse: 5'-atcgcgcgcggtgtcatctatgtt-3'; Fig. 1A).

\subsection{Regeneration and Selection on Media}

The transgenic plants were grown in soil to obtained T1 wheat seeds. In order to confirm the transgenic nature of the plants, the T1 seeds were soaked overnight in distil water and the embryos were removed aseptically. The excised embryos were placed on MS half media with $50 \mathrm{mg} / \mathrm{L}$ of kanamycin for selection.

\subsection{Nucleic acid isolation and PCR confirmation}

Plant genomic DNA will be extracted from $100 \mathrm{mg}$ in vitro plant material i.e. seeds using the CTAB (cetyltrimethylammonium bromide) method using DNA extraction buffer 1\% (w/v) SDS, 100mM NaCl, $100 \mathrm{mM}$ Tris base, $100 \mathrm{mM} \mathrm{Na}_{2}$ EDTA, $\mathrm{pH}=8.5$ by $\mathrm{HCl}$ ), as described earlier by Murray et al., (1980). PCR Primer (PGluB-4 Forward: 5'-gcacgccagaaaatataatgata-3'; TM-1 Reverse: 5'- cagtacgccgagcatact-3') were utilized to confirm the transgene integration. The Polymerase Chain Reactions (PCR) analysis was conducted for the presence of DNA encoding vaccine antigen. Specific primers to promoter region and TM-1 gene amplified the sequences in transgenic wheat seeds tissues. The DNA extracted from transgenic wheat seeds was used as a template for PCR analysis. The amplification for TM- 1 was carried out at $94^{\circ} \mathrm{C}$ for 5 $\min , 94^{\circ} \mathrm{C}$ for $1 \mathrm{~min}, 61^{\circ} \mathrm{C}$ for $1.5 \mathrm{~min}$ and $72^{\circ} \mathrm{C}$ for $1 \mathrm{~min}$, with total of 35 cycles, and final extension of $72^{\circ} \mathrm{C}$ for $10 \mathrm{~min}$. The amplified PCR product was resolved at 1\% SDS gel electrophoresis and was finally analyzed under UV light to confirm the presence of estimated band size. After confirmation, all the positive plants were self-pollinated to obtain T1 generation and were utilized for all analysis.

\subsection{Recombinant Protein extraction}

To find out the accumulation of recombinant fusion proteins, $50 \mathrm{mg}$ of seed powder was used to mix with 500 $\mu$ of $N$-hexane followed by centrifugation for $5 \mathrm{~min}$ at $5000 \times \mathrm{g}$, and supernatant was discarded. The process is repeated 3 times and fine seed powder was dried in vacuum. Double $\mathrm{dH}_{2} \mathrm{O}$ was added to powder with $1 / 10(\mathrm{w} / \mathrm{v})$ seed weight/water ratio and were incubated for 1 hour with agitation, followed by centrifugation of $13000 \mathrm{rpm}$ at $4^{\circ} \mathrm{C}$. The samples was homogenized with 3 volume of protein extraction buffer $(3 \%(\mathrm{w} / \mathrm{v})$ CTAB, 1,4 M NaCl, 0,2 \% (v/v) ß-Mercaptoethanol, 20 mM EDTA, pH 8.0, 100 mM Tris-HCl, pH 8.0, 1 $\%(\mathrm{w} / \mathrm{v})$ PVP40). Centrifuge for $15 \mathrm{sec}$ and the 100ul of supernatant was added to new 1.5 Eppendorf tube. Centrifuge again for $40 \mathrm{~min}$ at $13,000 \mathrm{rpm}$ at $4^{\circ} \mathrm{C}$. The supernatant will be discarded, and the pellet will be re-suspended in $5 \mathrm{X}$ loading buffer for further analysis. The total soluble protein (TSP) concentration of the extracts was determined by Bradford assay.

\subsection{SDS-PAGE and western blot analysis}

The total protein extracted from transgene positive wheat seeds tissues was then separated by SDS-PAGE on their molecular masses. The expression of vaccine antigen in seeds was verified by western blot analysis. The band were separated using $8 \%$ gel and were incubated with PBST containing $5 \%$ skimmed milk for one hour. The blot was then incubated with primary with 1:10000 ration with polyclonal rabbit anti-CTB antibody (Sigma-Aldrich, Inc,) over night at $4^{\circ} \mathrm{C}$ with continuous shaking. The blot was then washed three 
times with PBST and then was incubated with secondary goat anti-rabbit IgG-HRP antibody (Southern Biotechnology, Birmingham, AL) with ratio 1:5000 in PBST containing 5\% skimmed milk. The expected bands corresponding to TM-1 protein was detected by substrate NBT/ BCIP and developed with X-Ray for 5 seconds exposure. The quantitation of specific band was performed using software ImageJ.

2.6 ELISA quantification Enzyme-linked immunosorbent assay (ELISA) was performed using Pierce Nickel Coated Plates (Thermo Fisher Inc.) to detect the expression of TM-1 recombinant vaccine in wheat seeds by following manufacture's instruction. All the plant samples were analyzed in duplicate. For detection of antibody titer in immunized chickens, chickens were bled, and the serum was analyzed by the ELISA against GM antibody (Idexx Laboratories) following manufacture's instruction.

\subsection{Immunization and challenge procedure}

We included 100 two-days-old chicks separated randomly into four groups of 25 chicks each. Three groups were vaccinated orally with seeds powder containing $15 \mu \mathrm{g}$ recombinant TM-1 protein, same amount of WT seeds powder, and equal amount of PBS (Phosphate-buffered saline) buffer twice at day 7 and 21 . The fourth group was inoculated with MG attenuated vaccine $\left(8 \times 10^{6} \mathrm{CFU}\right)$ via ocular method. MG virus S6 strain $\left(5 \times 10^{6} \mathrm{CFU}\right)$ challenge was conducted through eye route for the all groups at week 4 and 5 . Clinical parameters were recorded daily after challenge with MG virus strain as described previously for two weeks [18]. Dead chickens before termination of the study were necropsied. All others were euthanized and examined two weeks after MG challenge. Briefly, the main clinical scores consisted of four levels in terms of symptomatic severity as follows: 0 - no symptom; 1 - clear nasal exudate; 2 - cloudy nasal exudate; 3 - turbid nasal exudate with foamy eyes and/or swollen infra-orbital sinuses. For air sac lesion, scores were observed and examined at 14 days after challenge as follows [19]: 0 - no lesions; 1 - mild lesions; 2 - moderate lesions; and 3 - severe lesions. Individual bodyweight was also recorded every week. All experiment groups were challenged with MG virulent strain $\left(5 \times 10^{6} \mathrm{CFU}\right)$ one week after second immunization by ocular delivery, followed by recording clinical parameters for additional two weeks.

2.8 Statistical analysisThe statistical analysis of this study was carried out using the one-way ANOVA analysis and indicated by $P$ value.

\section{Results}

\subsection{Creation and characterization of transgenic wheat seeds expressing recombinant TM-1.}

Lower expression of plant made biopharmaceutical is a great concern and hurdle for its commercialization. Several efforts including enhanced promoters, regulatory elements, protein targeting, utilization of various plants and their tissues and codon optimizing improve overall yield. It has been known that mRNA stability and translational efficiency are enhanced by codon optimization due to idealized tRNA usage preference of recombinant TM-1 biosynthesis in host cells [20]. Therefore, coding sequence of TM-1 was optimized during which 88 out of 262 codons in native sequence were replaced, resulting in an elevated GC\% from $31.93 \%$ to $46.44 \%$ (supplementary Fig. S1). The rice seed-specific promoter GluB-4 was included in construct as it can drive and target recombinant protein in rice endosperm with the highest translational activity among other 14 rice seed-specific promoters [17] (Fig. 1A). The ER retention signal peptide KDEL linked to 3' end of TM1 coding sequence has been shown to dramatically increase recombinant protein expression in rice endosperm [21]. CTB fused immediately upstream of TM-1 allows transport of TM-1 through gastrointestinal epithelia to circulation system by binding to a mucosal membrane receptor ganglioside GM1 [20]. Furin cleavage site can be digested by furin, a ubiquitous protease in human cells, enabling release of TM-1 after transmucosal transfer carried by CTB [22].

Three independent transgenic wheat lines were generated with particle bombardment and its subsequent regeneration and selection, and the line with highest expression was selected for further analysis. To verify the insertion of CTB-TM-1 construct in wheat genome, PCR with construct-specific primers was performed using genomic DNA from wheat T1 transgenic endosperm (Fig. 1B). The presence of CTB-TM-1 construct was detected in all three lines (Fig. 1B). The Western blot further confirmed the presence of recombinant 
TM-1 in wheat seeds from all lines with mean expression level of $0.84 \pm 0.046,1.03 \pm 0.068$, and $0.92 \pm 0.096$ $\mathrm{mg} / \mathrm{g}$ dry weight (DW) respectively and correct size as expected ( $\sim 1 \mathrm{kDa})$ (Fig. 1C and 1D). To evaluate the proper formation of CTB pentamer that binds GM1 receptor, a GM1 ELISA assay was carried out with crude extract of wheat seeds expressing recombinant CTB-TM-1. The results showed CTB-TM-1 correctly bond to GM1 in vitro when compared to CTB standard (Fig. 1E). In addition, unless otherwise indicated, the T1 seeds of line TM-1-2 was used for all downstream analysis and processes due to its highest TM-1 expression level and prominent affinity to GM1 receptor.

The transgenic nature of wheat plants transformed with CTB-TM-1 cassette by biolistics were tested on MS $\frac{1}{2}$ media containing antibiotic as selection marker. All the embryo excised from PCR confirmed transgenic T0 plants showed efficient regeneration as compared to wild type embryos as negative control. Total of 25 embryos excised from transgenic wheat line were confirmed by transgene integration and showed regeneration (Fig. 2).

\subsection{Stability, functionality, and immune response is preserved after storage for 6 months}

Plant seeds has natural ability to protect the vaccine antigen by bioencapsulation in the plant tissues, and therefore allow its storage at ambient temperature, moisture, and light conditions, and thus simplifies its production and distribution. We have tested our plant made vaccine antigen for confirmation of immunogenicity after long-term storage. The immunoblot (Fig. 3) confirmed TM-1 vaccine antigen can be stored at ambient temperature without degradation for at least six months. The confirmation of immune response is evident of such bioencapsulation where vaccine antigen was intact during passing through digestive system. Cell wall mediated bioencapsulation allow proteins to withstand enzymatic condition and can reach the small intestine, where the TM-1 vaccine antigen was incorporated into M cells in the follicle-associated epithelium (FAE), and finally to systemic immune responses. The CTB is FDA approved as fusion protein for delivery of biopharmaceuticals into immune system. The furin sites are incorporated between CTB and TM1 antigen for its delivery upon its cleavage. In addition, such results are encouraging because this protection to withstand biodegradation elements and stability at normal room conditions would allow plant made vaccine to eliminate the moderate to ultra-cold conditions required for vaccine storage and distribution. Also, we confirmed the feasibility of oral delivery of plant made vaccine production and its efficacy for animals with efficient delivery vehicle such as CTB and cleavage sites

\subsection{Immune response to vaccination of recombinant TM-1}

To evaluate the humoral immune response to vaccination of recombinant TM-1, serum from vaccinated chickens were analyzed with ELISA against anti-MG antibody weekly starting from the first week after the initial vaccination. The titer of anti-MG antibody started to increase in TM-1 and MG attenuated vaccine groups one week after initial immunization, although not significantly higher compared to control groups, until two weeks later (Fig. 4). Moreover, while MG attenuated vaccine caused higher anti-MG antibody titer, it is not significantly different from recombinant TM-1 group (Fig. 4). Notably, amount of anti-MG antibody was improved even more one week after second vaccination, leading to a greater significance compared to control group vaccinated with WT and PBS buffer (Fig. 4).

\subsection{Immunization of recombinant TM-1 protected chicken against MG infection.}

We next tested if twice vaccination could adequately protect chickens from MG infection by monitoring change of clinical sings and bodyweight after MG virulent strain inoculation. After two weeks of challenge, the mean severity-based clinical scores in control and WT group was $2.73 \pm 0.28$ and $2.65 \pm 0.26$, which were significantly higher than groups vaccinated with recombinant TM-1 and MG attenuated vaccine with a score of $0.72 \pm 0.13$ and $0.58 \pm 0.19$ respectively (Fig.5), indicating an efficient systemic protection against MG virus infection. Additionally, Bodyweight remained similar among all groups up to week 5 , suggesting vaccination of recombinant TM-1 antigen and attenuated MG vaccine did not affect gaining weight (Table 1). Nevertheless, chickens in TM-1 and attenuated MG vaccine groups gained significantly more weight than those in control group one week after MG virus challenge (Table 1), confirming the protective effect of recombinant TM-1 antigen prior to MG infection. Scores of air sac lesion showed similar pattern. After two weeks of MG virus 
challenge, presence of lesions was found significantly more in control groups compared to other two groups, suggesting the recombinant TM-1 vaccine specifically protects air sac in avian respiratory system (Table 2).

\subsection{Clinical signs}

In all groups, no adversary vaccine reaction was observed at administration site. Also, there were no clinical symptoms, apparent phenotype alteration or mortalities were recorded in any bird after challenge exposure that also includes all non-vaccinated controls. The birds were also monitored for its fertility and did not cause any fertility issue in all birds we studied.

\section{Discussion}

In chickens, Mycoplasma gallisepticum is the primary etiological agent of CRD. MG as major respiratory pathogen of poultry that causes severe inflammatory response in the trachea of its host [23]. The pathogen leads to develop clinical symptoms such as ocular, sneezing and coughing etc. and in severe cases causes pneumonia, bronchitis and airsacculitis in chickens and turkeys [24]. After infection, MG resides on mucosal surface, and causes epithelial cell degeneration and also result in inflammatory cell infiltration into the mucosa. The infectious disease affecting poultry industry that accounts for huge financial loses by drastically reducing productivity. Besides, lower production, commercially available vaccines are administered to control the severity also add to additional costs. The MG infection control is achieved by maintaining disease free breeding flock [25] and also by both in inactive and live attenuated vaccines. The commercially available vaccine such as strain 6/85 [26] F strain [25] and ts-11are applied for disease control, however they are expensive and require cold chain for storage and distribution.

The availability of vaccines for chronic respiratory disease in chickens are limited and expensive. Producing vaccine using conventional production system such as microbial or animal cell culture-based system are prohibitively expressive because of associated maintenance, storage, distribution and safety that accounts for $80 \%$ higher production cost in comparison to plant-based system. Plants have natural ability to store numerous proteins in seed tissues are excellent destination for production of recombinant proteins [27]. Numerous proteins have been targeted into seed tissue, where the target proteins showed stability as well as functionality several months after storage at ambient temperature [10]. The vaccine candidates as expressed in plant tissues induced the immunogenic responses has encouraged this study to express vaccine candidate against CRD in chickens in cost effective manner.

In addition, in poultry industry, in developing world, the more threatening is that chickens are supplied with numerous antibiotics to control different infectious diseases. In addition, the residue of such antibodies has been found in chickens which are directly consumed by the humans could be threatening for their health. Therefore, the promotion of alternative production systems for cost effective vaccine development is required to ensure access to modern medicine and improve health particularly in developing world.

In this study, the construct seed specific promoter was incorporated to induce TM-1 expression in seed tissues to achieve goal of oral delivery. The integration of an expression cassette carrying transgene into wheat developing callus was achieved by stable transformation biolistic. The TM- 1 protein was governed by a rice endosperm-specific promoter $G l u B-4$ with focus on seed tissues. Although, the fusion protein was also detected in vegetative tissues, however, such data was not included in this manuscript, and achieving goal of oral delivery of seed-based system is reported and discussed. The seed-based system is favored because such tissues are natural production and accumulation tissues for numerous proteins. Taking advantage of such ability we targeted our candidate vaccine antigen to achieve higher expression and its bioencapsulation. Seeds are relatively lower in water (10-40\%) in comparison to other plant tissues where water contents are up to $90 \%$ of their wet weight [28]. Thus, plant seeds are ideal vehicle with lower water content with denser proteins concentration which favors oral delivery of vaccines. Taking advantage of seed tissues, several vaccine antigens and antibodies showed prolonged stability for years without loss of activity at normal room conditions [28, 29].

The oral delivery for PDs is novel method for developing edible vaccine and has been reported earlier in 
several studies [14-16, 30-34]. The oral delivery of PDs does not necessitate the downstream processing which accounts for are more than $80 \%$ of total production cost. The cold chain requirement for vaccine storage and delivery are the major concerns to equitably vaccinate the resource poor population in developing countries where the average daily income is less than $\$ 2$. In the developing world, children die because of the unavailability of vaccines against life threatening infectious diseases. In this scenario, availability of vaccine to against veterinary disease is very unlikely. This is because maintaining cold chain without continuous electricity supply for its storage and distribution is near to impossible. Likewise, poultry industry is heavily affected by zoonotic diseases where high cost and unavailability of vaccines are major concerns, and those if available are costly and require cold temperature.

In this study, we demonstrated the oral route for vaccine delivery which is promising alternative to drastically reduce the cost of production, where vaccine candidate does not require further purification steps. Due to plant cell wall natural ability of bioencapsulation of the vaccine antigen to eliminate the probability of its degradation by providing protection from enzymatic conditions and preserved their antigen immunogenicity and functionality in the gastrointestinal tract (The unique characteristic of plant cells is their ability to withstand the digestive enzymes as present in humans and animals. The presence of certain bacteria in small intestine has the ability to break the plant cell wall to release the vaccine and are absorbed. The successful oral delivery of recombinant TM-1 antigen produced in wheat seeds was evidenced by results showing dramatically increased anti-MG antibody titer in chicken sera after vaccination compared to those vaccinated with WT and PBS buffer (Fig. 4). This was further supported by the observation that oral vaccination of recombinant TM-1 antigen efficiently protected chickens from MG infection later, while chickens in control groups were greatly infected and developed corresponding clinical symptoms (Fig. 5, Table 2).

To date, there are fewer plant made vaccine and monoclonal antibody candidates received regulatory authorization. Plant made products, i.e. poultry vaccine against NDV and antibody against HBsAg are the front runners in 30 years long down the road journey of molecular farming. The NDV vaccine is produced in suspension culture of tobacco cell, however, there is no oral product so far in market for orally delivered plant made vaccine. The oral delivery of plant made vaccine antigen received publicity and attention for their ease of delivery and cost effectiveness. This study is indeed can be as precedent for oral delivery of vaccine antigens against animal diseases where seed-based tissue showed decent expression efficacy, immunogenicity, and stability.

\section{Concluding remarks}

This study presents a proof of concept of producing oral vaccine in wheat seeds against Chronic Respiratory Disease (CRD) in poultry. The recombinant CTB-TM-1 protein of Mycoplasma gallisepticum (MG) expressed in transgenic wheat seeds remained stable and functional after long-term storage at ambient temperature. In addition, oral delivery of CTB-TM-1 vaccine bioencapsulated in wheat seeds successfully induced immune responses and protective effect against MG challenge in chicken without sacrifice of healthy development. Thus, this study demonstrates the feasibility, simplicity, and efficiency of seed-bioencapsulated oral vaccine in treating zoonotic infectious diseases.

\section{Acknowledgments}

We acknowledged International Islamic University for providing wildtype wheat plants for creation of transgenic lines, as well as animals and facilities necessary for immunization experiments used in this study.

\section{Conflict of interest statement}

The authors declare no commercial or financial conflict of interest

Funding : This work was supported by the Higher Education Commission of Pakistan (HEC). Project number: NRPU10635.

Table 1.

Weekly bodyweight change 


\begin{tabular}{lllllll}
\hline Group & Bodyweight $(\mathrm{kg})$ & Bodyweight $(\mathrm{kg})$ & Bodyweight $(\mathrm{kg})$ & Bodyweight $(\mathrm{kg})$ & Bodyweight $(\mathrm{kg})$ & $\mathrm{I}$ \\
\hline & Week 0 & Week 1 & Week 2 & Week 3 & Week 4 & \\
PBS & $0.09 \pm 0.01^{\mathrm{a}}$ & $0.15 \pm 0.01^{\mathrm{a}}$ & $0.34 \pm 0.03^{\mathrm{a}}$ & $0.64 \pm 0.03^{\mathrm{a}}$ & $0.96 \pm 0.06^{\mathrm{a}}$ & \\
WT & $0.09 \pm 0.01^{\mathrm{a}}$ & $0.16 \pm 0.01^{\mathrm{a}}$ & $0.35 \pm 0.03^{\mathrm{a}}$ & $0.66 \pm 0.03^{\mathrm{a}}$ & $0.96 \pm 0.07^{\mathrm{a}}$ & 1 \\
TM-1 & $0.09 \pm 0,01^{\mathrm{a}}$ & $0.16 \pm 0.01^{\mathrm{a}}$ & $0.35 \pm 0.02^{\mathrm{a}}$ & $0.64 \pm 0.04^{\mathrm{a}}$ & $0.94 \pm 0.06^{\mathrm{a}}$ & \\
MG attenuated vaccine & $0.09 \pm 0,01^{\mathrm{a}}$ & $0.15 \pm 0.01^{\mathrm{a}}$ & $0.36 \pm 0.02^{\mathrm{a}}$ & $0.65 \pm 0.04^{\mathrm{a}}$ & $0.97 \pm 0.08^{\mathrm{a}}$ & 1 \\
\hline
\end{tabular}

Note: The superscript letters $(\mathrm{a}, \mathrm{b})$ represent date from groups that were statistically significant $(P<0.05)$ scales when compared to each other.

Table 2.

Air sac lesion scores.

\begin{tabular}{lll}
\hline Group & Chicken number $(\mathrm{N})$ & Air sac lesion scores \\
\hline PBS & 25 & $2.6(0,3)^{\mathrm{a}}$ \\
WT & 25 & $2.5(0,3)^{\mathrm{a}}$ \\
TM-1 & 25 & $0.7(0,2)^{\mathrm{b}}$ \\
MG attenuated vaccine & 25 & $0.5(0,1)^{\mathrm{b}}$ \\
\hline
\end{tabular}

Note: The superscript letters $(\mathrm{a}, \mathrm{b})$ represent date from groups that were statistically significant $(P<0.05)$ scales when compared to each other.

\section{References}

[1]. Knödler M., Buyel J.F., Plant-made immunotoxin building blocks: A roadmap for producing therapeutic antibody-toxin fusions. Biotechnol Adv . 202147 :107683. doi: 10.1016/j.biotechadv.2020.107683.

[2]. Stoger E., Ma J. K., Fischer R., Christou P., Sowing the seeds of success: pharmaceutical proteins from plants. Curr Opin Biotechnol . 2005;16(2): 167-173. doi:10.1016/j.copbio.2005.01.005

[3]. Fischer, R., Twyman, R. M., and Schillberg, S. Production of antibodies in plants and their use for global health. Vaccine , 2003, 21 , 820-825. doi: 10.1016/s0264-410x(02)00607-2.

[4]. Mikschofsky, H., König, P., Keil, M., Hammer, M., et al. Choleratoxin B (CTB) is functional as an adjuvant for cytoplasmatic proteins if directed to the endoplasmatic reticulum (ER), but not to the cytoplasm of plants. Plant Sci , 2009, 177, 35-42.

[5]. Ceballo Y., Tiel K., López A., Cabrera G., et al. High accumulation in tobacco seeds of hemagglutinin antigen from avian (H5N1) influenza. Transgenic Res . 2017, 26(6) :775-789. doi: 10.1007/s11248-017-0047-9.

[6]. Shahid, N., Samiullah, T. R., Shakoor, S., Latif, A., et al. Early Stage Development of a Newcastle Disease Vaccine Candidate in Corn. Front Vet Sci , 2020, 7 , 499. doi: 10.3389/fvets.2020.00499

[7]. Salem R., Assem S. K., Omar O. A., Khalil A. A., et al. Expressing the immunodominant projection domain of infectious bursal disease virus fused to the fragment crystallizable of chicken IgY in yellow maize for a prospective edible vaccine. Mol Immunol . 2020,118 :132-141. doi: 10.1016/j.molimm.2019.12.015.

[8]. Vermij P. USDA approves the first plant-based vaccine.Nat Biotechnol , 2006, 24 , 233-234.

[9]. Kanci A., Wijesurendra D. S., Wawegama N. K., Underwood G. J., et al. Evaluation of Mycoplasma gallisepticum (MG) ts-304 vaccine as a live attenuated vaccine in turkeys. Vaccine . 2018,36(18 ):2487-2493. doi: $10.1016 /$ j.vaccine.2018.02.117. 
[10]. Khan I., Twyman R. M., Arcalis E., Stoger E., Using storage organelles for the accumulation and encapsulation of recombinant proteins. Biotechnol J . 2012, 7(9): 1099-108. doi: 10.1002/biot.201100089.

[11]. Arcalis E., Ibl V., Peters J., Melnik S., et al. The dynamic behavior of storage organelles in developing cereal seeds and its impact on the production of recombinant proteins. Front Plant Sci . 2014,3;5 :439. doi: $10.3389 /$ fpls.2014.00439.

[12]. Hudson L. C., Garg R., Bost K. L., Piller K. J., Soybean seeds: a practical host for the production of functional subunit vaccines. Biomed Res Int . 2014: 340804. doi: 10.1155/2014/340804.

[13]. Vamvaka E., Arcalis E., Ramessar K., Evans A., et al. Rice endosperm is cost-effective for the production of recombinant griffithsin with potent activity against HIV. Plant Biotechnol $J$. 2016, 14 (6): 1427-37. doi: 10.1111/pbi.12507.

[14]. Park Y., An D-J., Choe S., Lee Y., et al. Development of recombinant protein-based vaccine against classical swine fever virus in pigs using transgenic Nicotiana benthamiana. Front Plant Sci . 2019, 10 , 624. doi: $10.3389 /$ fpls.2019.00624

[15]. Reddy P. H., Johnson A. A., Kumar J. K., Naveen T., et al. Heterologous expression of Infectious bursal disease virus VP2 gene in Chlorella pyrenoidosa as a model system for molecular farming. Plant Cell Tissue Organ Culture, 2017, 131 , 119-26. doi: 10.1007/s11240-017-1268-6

[16]. Gunter CJ., Regnard GL., Rybicki EP., Hitzeroth II. Immunogenicity of plant produced porcine circovirus-like particles in mice. Plant Biotechnol J , 2019, 17 , 1751-9. doi: 10.1111/pbi.13097

[17]. Qu L. Q., Takaiwa F., Evaluation of tissue specificity and expression strength of rice seed component gene promoters in transgenic rice. Plant Biotechnol J . 2004, 2(2): 113-25. doi: 10.1111/j.1467-7652.2004.00055.x.

[18]. Naylor, C.J., Al-Ankari A.R., Al-Afaleq, A.I., Bradbury, J.M. et al. Exacerbation of Mycoplasma gallisepticum infection in turkeys by rhinotracheitis virus. Avian Pathol , 1992, 21 , 295-305. doi: $10.1080 / 03079459208418844$.

[19]. Ganapathy, K. \& Bradbury, J.M. Pathogenicity of mycoplasma imitans in mixed infection with infectious bronchitis virus in chickens.Avian Pathol , 1999, 28 , 229-237. doi: 10.1080/03079459994713.

[20]. Takaiwa F., Wakasa Y., Takagi H., Hiroi T. Rice seed for delivery of vaccines to gut mucosal immune tissues. Plant Biotechnol J . 2015, 13(8): 1041-55. doi: 10.1111/pbi.12423.

[21]. Takaiwa F., Yang L., Wakasa Y., Ozawa K. Compensatory rebalancing of rice prolamins by production of recombinant prolamin/bioactive peptide fusion proteins within ER-derived protein bodies. Plant Cell Rep . 2018 37(2 ):209-223. doi: 10.1007/s00299-017-2220-2.

[22]. Thomas G. Furin at the cutting edge: from protein traffic to embryogenesis and disease. Nat Rev Mol Cell Biol . 2002,3(10): 753-66. doi: 10.1038/nrm934.

[23]. Haycraft, C. J., Schafer, J. C., Zhang, Q., Taulman, P. D., et al. Identification of CHE-13, a novel intraflagellar transport protein required for cilia formation. Exp Cell Res. 2003;284(2): 251-263. doi:10.1016/S00144827(02)00089-7

[24]. Nunoya T., Yagihashi T., Tajima M., and Nagasawa Y., Occurrence of keratoconjunctivitis apparentl,y caused by Mycoplasma gallisepticum in layer chickens. Vet Pathol. ,1995, 32 , 11-18. doi:10.1177/030098589503200102.

[25]. Levisohn S., and Kleven S. Avian mycoplasmosis (Mycoplasma gallisepticum). Rev Sci Tech . 2000;19(2 ):425-442. doi:10.20506/rst.19.2.1232.

[26]. Evans R., and Hafez Y., Evaluation of a Mycoplasma gallisepticum strain exhibiting reduced virulence for prevention and control of poultry mycoplasmosis. Avian Dis. 1992, 36 , 197-201. doi:10.2307/1591490 
[27]. Ramessar, K., Capell, T., and Christou, P.. Molecular pharming in cereal crops.Phytochemical Reviews 2008, 7, 579-592.

[28]. Boothe J., Nykiforuk C., Shen Y., et al. Seed-based expression systems for plant molecular farming Plant Biotechnol J . 2010,8(5): 588-606. doi: 10.1111/j.1467-7652.2010.00511.x.

[29]. Stoger E., Vaquero C., Torres E., Sack M., et al. Cereal crops as viable production and storage systems for pharmaceutical scFv antibodies. Plant Mol Biol . 2000, 42(4): 583-90. doi: 10.1023/a:1006301519427.

[30]. Shim B. S., Hong K. J., Maharjan P M., and Choe S. Plant factory: new resource for the productivity and diversity of human and veterinary vaccines. Clin Exp Vaccine Res , 2019, 8 , 136-9. doi: 10.7774/cevr.2019.8.2.136

[31]. Firsov A., Tarasenko I., Mitiouchkina T., Ismailova N., et al. High-yield expression of M2e peptide of avian influenza virus H5N1 in transgenic duckweed plants. Mol Biotechnol , 2015, 57 , 653-61. doi: 10.1007/s12033-015-9855-4

[32]. Rizwan H. M., Khan M. K., Iqbal Z., and Deeb F. Immunological and therapeutic evaluation of wheat (Triticum aestivum) derived betaglucans against coccidiosis in chicken. International Journal of Agricultural Biology, 2016, 18, 895-902. doi: 10.17957/IJAB/15.0182

[33]. Berinstein A., Vazquez-Rovere C., Asurmendi S., Gómez E., et al. Mucosal and systemic immunization elicited by Newcastle disease virus (NDV) transgenic plants as antigens. Vaccine , 2005,23 , 5583-9. doi: $10.1016 / \mathrm{j}$.

[34]. Yang M., Sun H., Lai H., Hurtado J., et al. Plant-produced Zika virus envelope protein elicits neutralizing immune responses that correlate with protective immunity against Zika virus in mice.Plant Biotechnol $J$. 2018;16(2):572-580. doi: 10.1111/pbi.12796

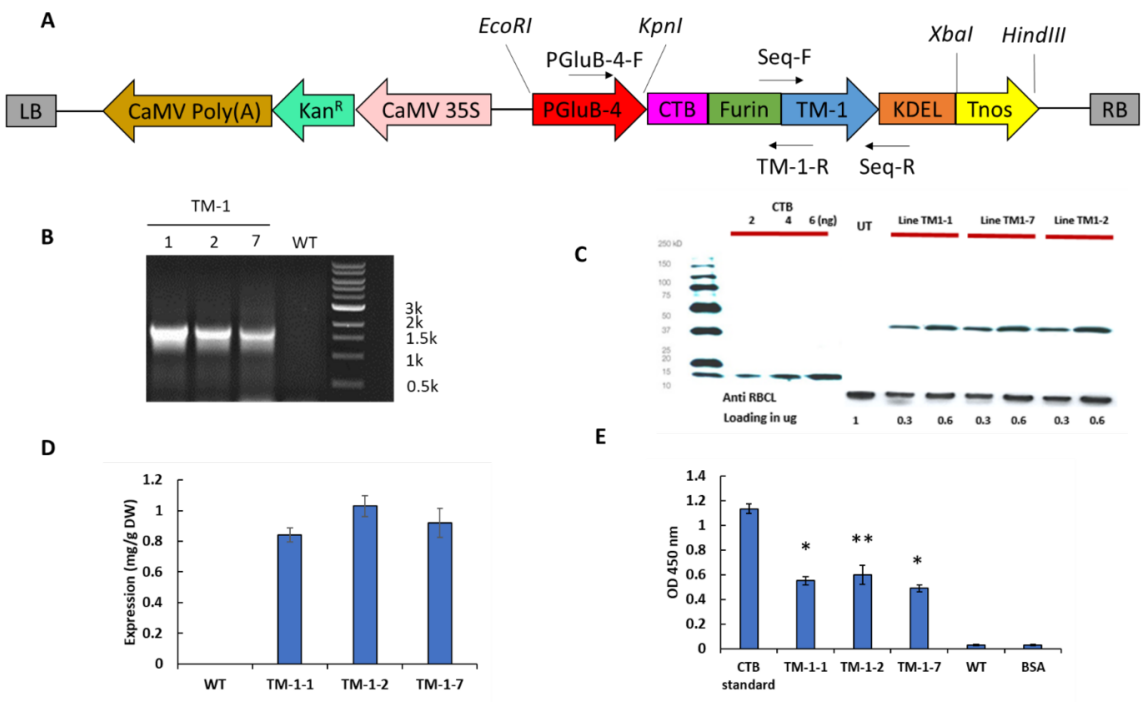

\section{Figures:}

Figure 1. Development and characterization of CTB-TM-1 expression cassette in transgenic wheat seeds. A. The schematic diagram of expression cassette of pPZP211: CTB-TM-1. Genetic components included are: PGluB-4, promoter of glutelin gene GluB-4; CTB, Cholera non-Toxin B subunit; Furin, Furin cleavage site; TM-1, TM-1 protein ofMycoplasma gallisepticum (MG); KDEL, Endoplasmic reticulum retention signal; Tnos, termination of the nopaline synthase gene fromAgrobacterium tumefaciens; CaMV 35S, promoter of 35S RNA from Cauliflower Mosaic Virus ; Kan ${ }^{\mathrm{R}}$, Kanamycin resistant gene; CaMV 
Poly(A), Cauliflower Mosaic Viruspolyadenylation signal; LB and RB, left and right border of T-DNA repeat respectively. Arrows indicate PCR and sequencing primers. B.characterization of genomic integration of pPZP211: CTB-TM-1 cassette in different transgenic lines by PCR using primers (CTB-F and TM-1R).C. Validation of three independent lines for recombinant CTB-TM-1 expression by Western blot with anti-CTB antibody. D.quantification of recombinant CTB-TM-1 expression in C. E. GM1 ELISA detecting the folding of CTB-TM-1 and its binding with GM1 receptor. ${ }^{*}, P<0.05,{ }^{* *}, P<0.01,{ }^{* * *}, P<0.001$.

A

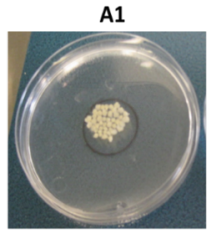

B

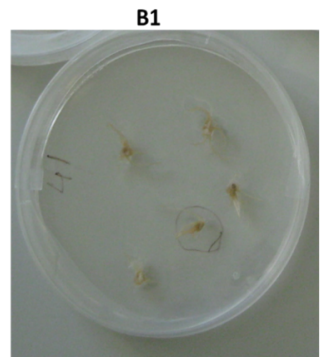

A2
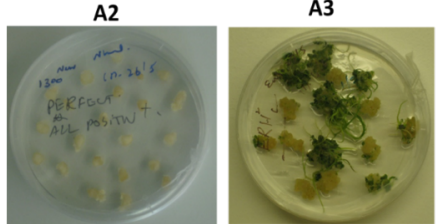

B2

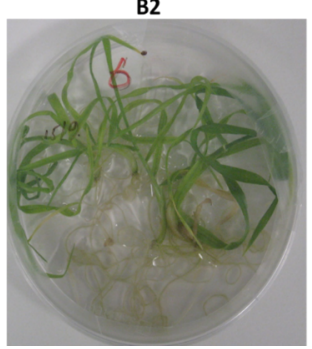

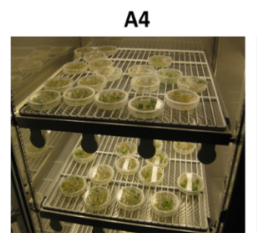

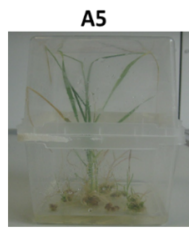

Fig. 2. Process of developing transgenic plant using Biolistics: Segregation of transgenic wheat lines : A1. Immature embryos after bombardment with plasmid carying TM-1. A2. Callus development under dark with antibiotic selection. A3. Plant regeneration in media containing selection marker. A4. Plant regeneration and rooting in growth chamber. A5. Wheat plant expressing TM-1 vaccine candidate in rooting media, before moving to soil for growth. B. Embryo rescue analysis. Wheat embryo excised from WT and positive plants and their germination on selection media. B. Embryo excision for transgene selection with kanamycine supplemented germinating media. B1. Wild type BobWhite as negative control. B2. Growth TM-1 positive embryo excised from seeds after 10 days on MS half media as control for the experiment.

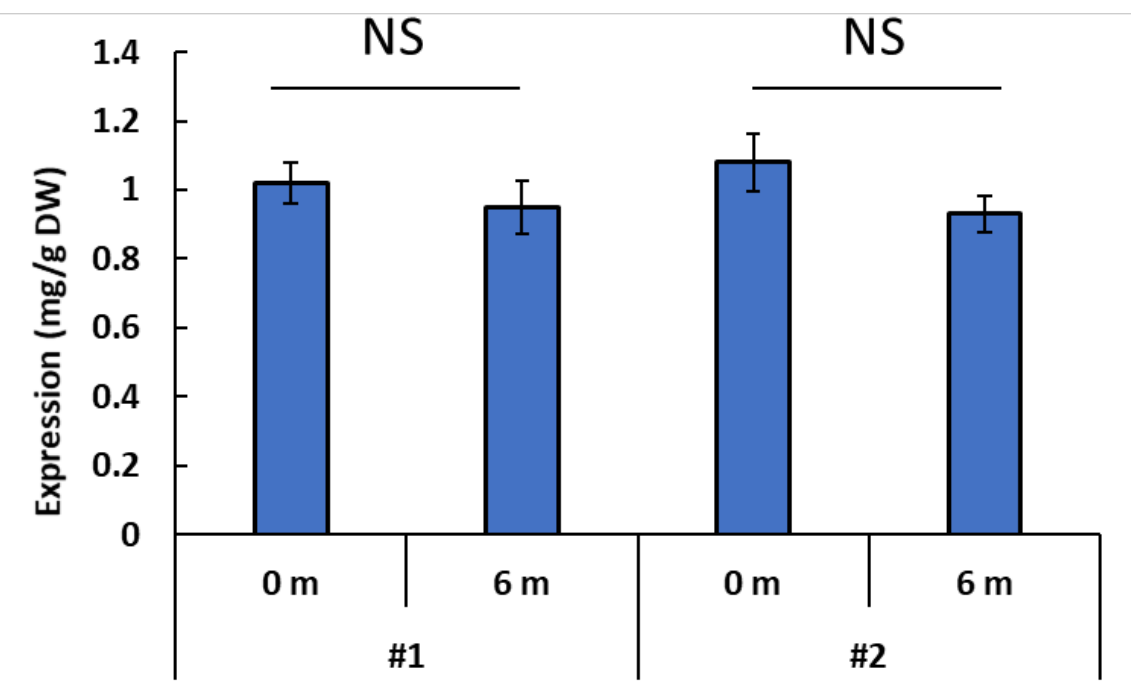


Figure 3. Stability of recombinant CTB-TM-1 expressed in wheat seeds after long-term storage at ambient temperature (transgenic line: TM-1-2). The recombinant CTB-TM-1 was extracted from seeds powder stored immediately after harvest and six months later as described in method and the expression level was quantified by western blot with anti-CTB antibody. NS: non-significant $(P>0.05)$

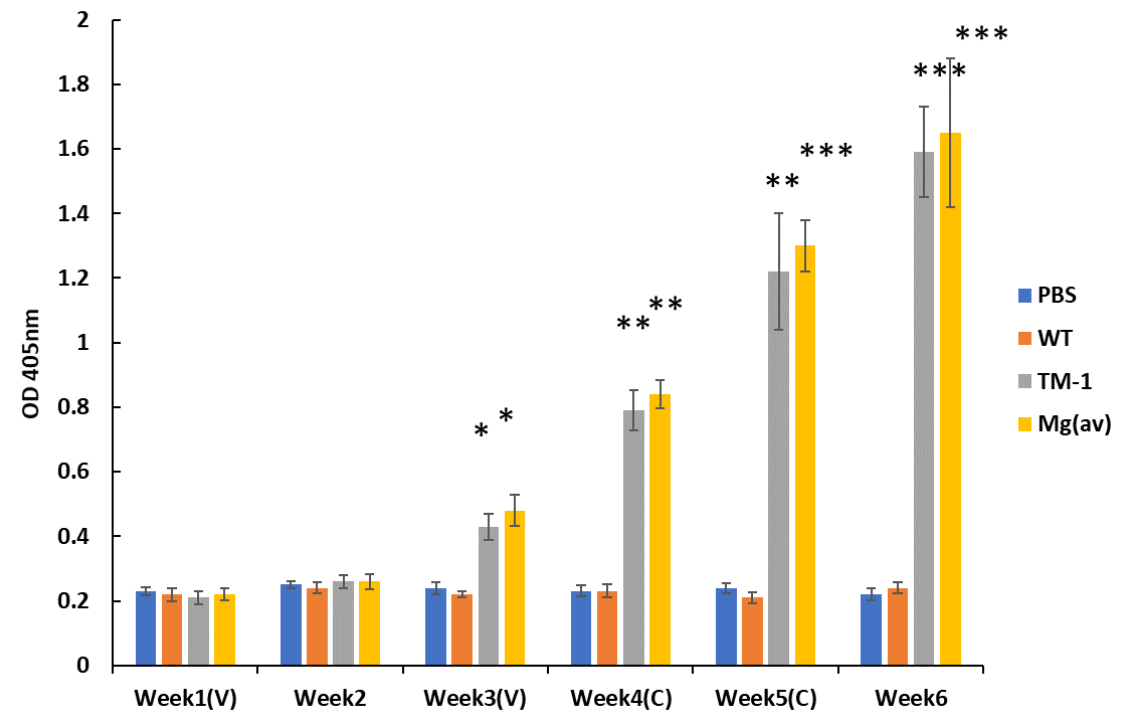

Figure 4. Immune responses to recombinant TM-1 vaccine. Anti-MG antibodies in chicken serum were quantified by ELISA after oral delivery of recombinant TM-1 vaccine, WT, and PBS except for group innoculated with attanuated $M G$ vaccine $[M G(a v)]$ by injection. $(\mathrm{V})$ and $(\mathrm{C})$ represent the week when vaccination or challenge occured respectively. ${ }^{*}, P<0.05,{ }^{* *}, P<0.01,{ }^{* * *}, P<0.001$.

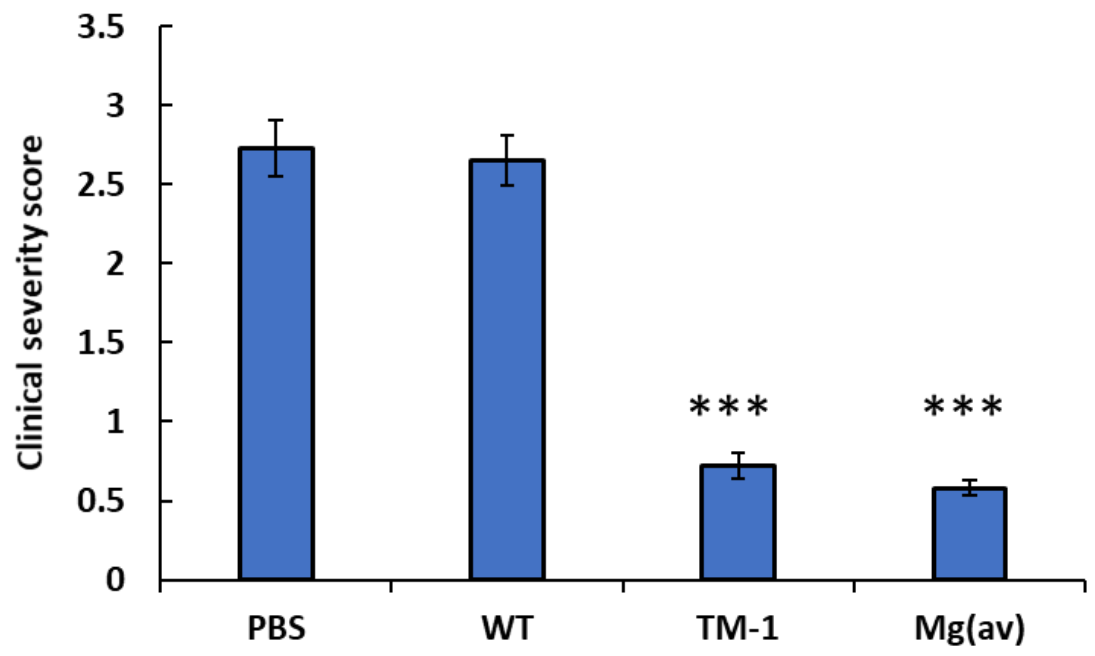

Figure 5. Protective effect of recombinant TM-1 immunization against MG challenge . Clinical severity was observed and recorded for two weeks after MG challenge. Score categories were assigned according to the description in materials and methods section in text. ${ }^{*}, P<0.05,{ }^{* *}, P<0.01,{ }^{* * *}, P<0.001$. 